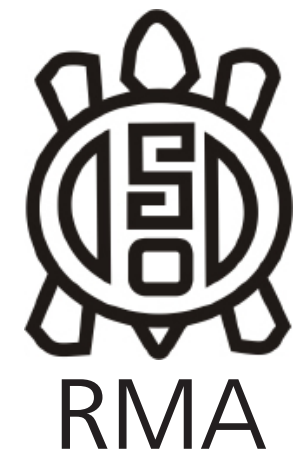

Antropología Biológica

\title{
Endogamia y consanguinidad en el Valle de Calamuchita, Provincia de Córdoba, Argentina. Período 1896-1950
}

\author{
Endogamy and inbreeding in the Calamuchita Valley, Córdoba Province, \\ Argentina. Period 1896-1950
}

Mayra Almeida* y Darío A. Demarchi**

* Universidad Nacional de Córdoba, Facultad de Filosofía y Humanidades, Departamento de Antropología, Córdoba, Argentina. E-mail: mayra_15a@hotmail.com

\begin{abstract}
**Instituto de Antropología de Córdoba, IDACOR, CONICET, Universidad Nacional de Córdoba, Córdoba, Argentina. Universidad Nacional de Córdoba, Facultad de Filosofía y Humanidades, Museo de Antropología, Córdoba, Argentina. E-mail: demarchi@ffyh.unc.edu.ar
\end{abstract}

\begin{abstract}
Resumen
En este trabajo se analizan cambios en la estructura de la población del Valle de Calamuchita durante el período 1896-1950 a partir de datos biodemográficos. La muestra incluyó la totalidad de los matrimonios registrados en el Registro Civil de Amboy, donde se anotan nacimientos, casamientos y defunciones de toda la población de Calamuchita desde 1896. A partir de datos de 1105 matrimonios, se estimó el coeficiente de consanguinidad por isonimia marital (F), los porcentajes de endogamia y de exogamia regional y extra regional y la distancia marital promedio. Los resultados revelan un alto grado de endogamia, salvo en la última cohorte (1940-1950) cuando comienza a observarse un mayor dinamismo migratorio en la población. Se obtuvieron valores de consanguinidad por isonimia relativamente altos, siendo el componente no aleatorio (Fn) muy superior al aleatorio (Fr) a lo largo de todo el período investigado, hecho que revela que se trata de una población consanguínea por elección y, en menor grado, debido a su pequeño tamaño efectivo. Este comportamiento puede ser explicado como consecuencia de una estrategia por mantener a resguardo el patrimonio familiar o bien por la preferencia conyugal por origen geográfico cercano.
\end{abstract}

Palabras Clave: Amboy; Apellidos; Biodemografía; Distancia marital; Migración.

\begin{abstract}
In this paper we analyzed changes in the population structure of the Calamuchita Valley during the period 1896-1950 based on biodemographic data. The sample included all the marriages registered in the Registro Civil of Amboy, where births, marriages and deaths of the entire population of Calamuchita have been recorded since 1896. Based on data from 1105 marriages, we estimated the coefficient of inbreeding by isonymy (F), the percentages of endogamy and regional and extra-regional exogamy and the average marital distance. The results reveal a high degree of endogamy, except in the last cohort (1940-1950) when a greater migratory dynamism begins to be observed in the population. High consanguinity values were obtained, the non-random component (Fn) being much higher than the random one (Fr) over the period under investigation, a fact that reveals that it is a consanguineous population by choice and, to a lesser extent, due to its small effective population size. This behavior might be explained as a consequence of a strategy to keep family patrimony safe or by the conjugal preference for close geographical origin.
\end{abstract}

Keywords: Amboy; Surnames; Biodemography; Marital Distances; Migration.

Una población puede ser estudiada desde la bioantropología tomando en cuenta los aspectos estrictamente genéticos, pero también atendiendo a aspectos demográficos de la misma. La evolución de las poblaciones humanas es resultado de la interacción entre factores biológicos, sus estructuras sociales, su cultura y el comportamiento de sus miembros. Los aspectos biológicos de reproducción, crecimiento y mortalidad se encuentran fuertemente ligados a complejas instituciones sociales, culturales, económicas, y en 
diversos y cambiantes espacios ecológicos y geográficos. (Dipierri, 2014). Además de los factores puramente biológicos que conducen a cambios microevolutivos, en las poblaciones humanas se suman una serie de elementos de carácter social y cultural, tales como los cruzamientos no aleatorios, que pueden conducir a la consanguinidad, la fecundidad preferencial de las parejas y las migraciones.

Los apellidos reflejan una parte importante de la identidad y de la estructuración de las sociedades. Su distribución en una población resulta de largos procesos históricos y culturales $y$, en consecuencia, representa una fuente de información valiosa para la reconstrucción de la historia cultural, así como para analizar, desde el punto de vista genético y demográfico, la estructura de la misma (Alfaro, 2010). Transmitidos generalmente por línea paterna, los apellidos funcionan como un locus con diferentes alelos, y su distribución en una población se asemeja a la de alelos selectivamente neutros bajo las fuerzas de migración y fluctuación génica (Barrai et al., 1991; Demarchi y Colantonio, 2000; Dipierri et al., 2005), no confiriendo ventajas selectivas a quienes los portan (Rodríguez Larralde, 1990). El modelo de variación de los apellidos se supone simple porque éstos no van asociados a ninguna diferencia en cuanto a fertilidad o mortalidad. Ahora bien, si la deriva y la migración provocan cambios en las frecuencias génicas de la población, éstas pueden atestiguarse en los cambios de las frecuencias de los apellidos (Lasker, 1991).

El término isonimia significa la ocurrencia del mismo apellido en dos individuos, la proporción con la cual ésta se presenta (Lasker, 1985) o la similitud en el apellido (Rawling, 1973). Quien propuso en primer término el método isonímico fue George Darwin (1875), hijo del naturalista Charles Darwin. Darwin se interesó en el posible efecto de deterioro que podría causar la consanguinidad en la descendencia y quiso saber la frecuencia de matrimonios entre primos hermanos en la Inglaterra del siglo XIX. Posteriormente, Crow y Mange (1965) desarrollaron desde la isonimia un método para calcular la consanguinidad de toda la población, que fue perfeccionado por Crow (1980). Este procedimiento permitió calcular parámetros biológicos de poblaciones de las que no se posee información genética. Aunque la estimación de la consanguinidad mediante isonimia es un método aproximado, puesto que gran parte de los apellidos tienen un origen polifilélico y no es raro que a lo largo del tiempo experimenten cambios, presenta ciertas ventajas que derivan de la facilidad de cálculo del coeficiente de isonimia y de la posibilidad de detectar la consanguinidad en generaciones más remotas que por medio de otros análisis genealógicos (Alfaro, 2010).

El acervo genético de las poblaciones del actual territorio de la Provincia de Córdoba es resultante de una larga secuencia de eventos históricos y evolutivos. Estos eventos no fueron homogéneos a través de todo el territorio, sino que estuvieron atravesados por distintos factores ambientales, políticos y económicos. Durante el período colonial, la población de la provincia se concentraba principalmente en la ciudad capital y en la región serrana, lugares que también fueron los de mayor densidad de población indígena en tiempos pre-hispánicos y donde estudios genéticos recientes encontraron las mayores proporciones de linajes maternos americanos y africanos (García et al. 2018). En contraste, la región de la llanura pampeana representa el área de ocupación más reciente y de menor densidad poblacional indígena y, junto con las grandes ciudades, el principal territorio de asentamiento de los migrantes europeos del período 1880-1950. Esto se refleja en una mayor proporción de linajes maternos euroasiáticos en las poblaciones del sur de Córdoba, como en las de otras localidades de la región pampeana (Pauro 2015). A partir de mediados del siglo XIX se inició en la Argentina una valorización de los suelos pampeanos en el marco de una incipiente economía agrícola-ganadera (Tell, 2008). Este cambio ocurrió poco antes de la llegada masiva de inmigrantes europeos (1880-1950) que se instalaron principalmente en las grandes ciudades y en los territorios pampeanos y patagónicos, como parte de la política del Estado argentino de colonización y "civilización" del territorio (Pauro, 2015).

El poblamiento reciente del Valle de Calamuchita, Provincia de Córdoba, resulta de particular interés dado que, en el imaginario de la mayoría de la población cordobesa, Calamuchita aparece como un espacio poblado de extranjeros de origen centro-europeo. Esto es particularmente notorio en la localidad de Villa General Belgrano, la cual, gracias a una estrategia destinada a la explotación turística, es percibida como una comunidad conformada a partir de migrantes alemanes.

En este artículo se aborda el estudio de la historia evolutiva reciente de la población del Valle de Calamuchita utilizando datos y herramientas de la Biodemografia. Su objetivo es investigar la evolución de la endogamia, la consanguinidad y el flujo génico en el período comprendido entre los años 1896 y 1950 a partir del análisis de las actas matrimoniales del Registro Civil de la localidad de Amboy.

\section{Población y Métodos}

\section{Lugar de estudio}

El Departamento Calamuchita es uno de los 26 departamentos en los que se divide la Provincia de Córdoba (República Argentina). Está ubicado en una posición ecotonal, entre las Sierra de Comechingones y la planicie suroriental, abarcando una superficie de $4.642 \mathrm{~km}^{2}$ donde viven 54.730 habitantes (INDEC, Censo 
2010). Se divide políticamente en siete pedanías donde se asientan cuarenta y cinco municipios y comunas. La comuna de Amboy, situada en la pedanía Cañada de Álvarez, se encuentra a 602 msnm y a $115 \mathrm{~km}$ al sudoeste de la ciudad de Córdoba. Aunque fue fundada oficialmente en 1896, históricamente es una de las poblaciones más antiguas de la provincia de Córdoba, con alrededor de 400 años de existencia. Con apenas 183 habitantes (INDEC, Censo 2010), cuenta con Registro Civil desde el año 1896, siendo éste el segundo más antiguo del Departamento. Allí se registran desde entonces nacimientos, casamientos y defunciones no sólo locales sino también de gran parte de la población del valle de Calamuchita.

\section{Fuente de datos}

Los datos para esta investigación fueron tomados de las copias de actas matrimoniales del Registro Civil de la comuna de Amboy que se encuentran en el Registro Provincial de las Personas de la Provincia de Córdoba, ubicado en la ciudad de Córdoba. La investigación se delimitó al período comprendido entre 1896 y 1950, coincidiendo esta última fecha aproximadamente con el final de la gran ola inmigratoria europea hacia Argentina. Se tomaron los siguientes datos de las actas matrimoniales: apellido y nombres de los cónyuges, nacionalidad, edad, profesión, lugar de nacimiento y lugar de residencia.

\section{Análisis biodemográfico}

A partir de la información sobre el lugar de nacimiento de los cónyuges se calcularon los siguientes parámetros de aislamiento poblacional:

1. Porcentaje de endogamia: obtenido teniendo en cuenta el número de matrimonios ocurridos entre dos personas nacidas en el Departamento Calamuchita.

2. Porcentaje de exogamia (Alfaro y Dipierri, 1996): obtenido teniendo en cuenta la cantidad de matrimonios con un cónyuge nacido en el Departamento Calamuchita y el otro no. Se consideran dos componentes:

a- exogamia regional: porcentaje de matrimonios con un contrayente nacido en la región central de Argentina;

b- exogamia no regional: porcentaje de parejas con un contrayente nacido fuera de esa región.

3. Índice de exogamia (Palatnik, 1975), estimado según la siguiente fórmula:

$$
\mathrm{e}=\frac{\% \text { de progenitores de otro lugar }}{\% \text { progenitores del lugar }}
$$

4. Distancia marital promedio (DMP). Obtenida como la distancia promedio que separa el lugar de nacimiento de los cónyuges. Las distancias (por rutas) se midieron en km (Araujo y Salzano, 1974). No se computaron las distancias maritales en los casos donde uno o los dos miembros de la pareja procedían de otro continente, ya que ese cómputo incrementaría desproporcionadamente el valor promedio.

A partir de los apellidos de los cónyuges, se estimó el grado de consanguinidad con el método de Isonimia propuesto por Crow y Mange (1965). Éste se basa en la relación constante de la probabilidad que tienen los parientes de cualquier grado de ser isónimos al compartir el mismo apellido heredado de un antecesor común. Esta probabilidad de isonimia (pi) es igual al coeficiente de consanguinidad (F) de sus hijos multiplicado por cuatro, de tal manera que pi/F es siempre igual a cuatro. En consecuencia, la frecuencia de parejas isónimas dividida por cuatro es el valor del coeficiente de consanguinidad de la población. Crow y Mange (1965) denominaron a este coeficiente F y posteriormente Crow (1980) lo dividió en dos fracciones: la fracción aleatoria $(\mathrm{Fr})$ y la fracción no aleatoria (Fn), proponiendo para ellas las siguientes fórmulas:

$\mathrm{Fr}=\left(\sum \mathrm{p}_{\mathrm{i}} \mathrm{q}_{\mathrm{i}}\right) / 4$,

donde $p_{i} q_{i}$ es la proporción relativa de cruzamientos entre personas con apellido i:

$F n=\left(P-\left(\sum p_{i} q_{i}\right) / 4\left(1-\left(\sum p_{i} q_{i}\right)\right.\right.$,

donde $\mathrm{P}$ es la proporción de isonimia;

$\mathrm{Ft}=\mathrm{Fn}+\mathrm{Fr}(1-\mathrm{Fn})$.

\section{Resultados}

En la Tabla 1 se presenta información sobre la totalidad de los matrimonios asentados en el Registro Civil de Amboy en el período seleccionado para esta investigación (1896-1950). El mismo fue dividido en seis cohortes con el fin de observar la posible variación en el comportamiento de los parámetros biodemográficos investigados a través del tiempo. En la primera cohorte, que abarca solamente 5 años, el $78 \%$ de los matrimonios se celebraron entre personas nacidas en el Departamento Calamuchita, mientras que el $18 \%$ fueron mixtos ${ }^{1} y$ el $3 \%$ foráneos $^{2}$. Los matrimonios mixtos y foráneos se celebraron con migrantes que procedían principalmente de la ciudad de Córdoba, Río Cuarto, San Javier y de la provincia de San Luis. Los registros muestran para este período 3 extranjeros, todos italianos, que se casaron con mujeres nacidas en Calamuchita. Dado el alto porcentaje de matrimonios locales, la distancia marital promedio observada es relativamente baja, menor a $45 \mathrm{~km}$. La segunda cohorte abarca desde 1901 hasta 1910, inclusive, y es la más numerosa del período estudiado. Del total de matrimonios, 80,7\% fueron locales, 16,9\% mixtos y $2,4 \%$ foráneos. La distancia marital fue levemente superior a la de la cohorte anterior, aunque la proporción de migrantes disminuyó levemente y los lugares de procedencia fueron similares (ciudad de Córdoba, Río Cuarto, San Javier y provincia de San

\footnotetext{
1 Uno de los cónyuges radicado en Calamuchita y el otro no.

2 Ninguno de los cónyuges de Calamuchita.
} 
Tabla 1. Matrimonios asentados en el Registro Civil de Amboy durante el período 1896-1950 y distancia marital promedio (DMP), por cohorte.

Table 1. Marriages registered at the Amboy Civil Registry during the period 1896-1950, and average marital distance (MCD), by cohort.

\begin{tabular}{|c|c|c|c|c|c|}
\hline & \multicolumn{4}{|c|}{ Matrimonios } & \\
\hline Período & Total & locales & mixtos & foráneos & DMP \\
\hline $1896-1900$ & 181 & 78,5 & 18,2 & 3,3 & 44,8 \\
\hline $1901-1910$ & 331 & 80,7 & 16,9 & 2,4 & 57,5 \\
\hline $1911-1920$ & 114 & 80,7 & 19,3 & 0 & 33,7 \\
\hline $1921-1930$ & 138 & 81,2 & 18,1 & 0,7 & 30,3 \\
\hline $1931-1940$ & 174 & 81,6 & 15,5 & 2,9 & 58,2 \\
\hline $1941-1950$ & 167 & 58,7 & 32,9 & 8,4 & 128,2 \\
\hline
\end{tabular}

Luis). Solamente se registraron dos migrantes extracontinentales, 2 hombres procedentes de Italia que contrajeron matrimonio con mujeres de Calamuchita. En la tercera y cuarta cohortes se observa una marcada reducción en el número de matrimonios anotados y en la distancia marital promedio. Este hecho puede deberse a la creación de 3 nuevos registros civiles en Calamuchita ${ }^{3}$ durante ese período. La proporción de matrimonios mixtos aumentó levemente pero solamente se registró una unión con ambos cónyuges foráneos. Se advierte ya un incremento de migrantes europeos que contrajeron matrimonio en este período, 4 españoles y un italiano en la tercera cohorte y 5 españoles, 2 italianos y un serbio en la cuarta. La quinta cohorte muestra la mayor proporción de matrimonios locales $(81,6 \%)$ pero, al mismo tiempo, un incremento en el número de matrimonios foráneos. También se observa un aumento en el valor de la distancia marital promedio, debido a la presencia de varios cónyuges procedentes de otras localidades de Córdoba y de la provincia de Santa Fe. Durante la quinta cohorte contrajeron matrimonio 7 migrantes extracontinentales, 5 procedentes de Italia y 2 de España. La última cohorte muestra una dinámica migratoria mucho más activa. Más del $40 \%$ de los matrimonios fueron mixtos o con ambos cónyuges procedentes del exterior del Departamento Calamuchita. Se observa también un incremento en la DMP, que duplica a las registradas en las cohortes anteriores. Debe tenerse en cuenta que no se computaron las distancias maritales en los casos donde uno o los dos miembros de la pareja procedían de otro continente, ya que este cómputo incrementaría desproporcionadamente este valor. Además de los migrantes de otros departamentos de Córdoba,

\footnotetext{
3 En el contexto histórico nacional ocurren una serie de hechos que explican la creación de diversos registros civiles en un corto período de tiempo. En el año 1911 y durante la presidencia de Roque Sáenz Peña, se dicta la ley nacional 8.129, o Ley de Enrolamiento militar y Padrón Electoral, que obliga a los ciudadanos argentinos a inscribirse en los nuevos Registros Civiles y obtener la libreta de enrolamiento (RENAPER 2019). El fin último de esta ley era tener un control y conteo de toda la población argentina a través de instituciones estatales, en una puja por eliminar los fraudes electorales y quitando exclusiva función para el registro de la población a la Iglesia Católica.
}

aparecen en este período cónyuges procedentes de las provincias de Formosa, Corrientes, Santa Fe, Buenos Aires y La Pampa. Los migrantes extranjeros fueron 18, 10 de España, 2 de Italia, 2 de Alemania, 1 de Paraguay, 1 de Polonia, 1 de Francia y 1 de Inglaterra.

En la Tabla 2 se presentan los porcentajes de endogamia y exogamia, el índice de exogamia de Palatnik y la proporción de migrantes por sexo (masculinos/femeninos). Los valores de endogamia oscilan entre $78,5 \%$ en la primera y $81,6 \%$ en la quinta, por lo que la exogamia es relativamente baja, tanto en sus componentes regional como no regional. En la última cohorte disminuye marcadamente la endogamia y se duplica el porcentaje de exogamia regional. Sin embargo, el porcentaje de exogamia no regional no refleja la mayor afluencia de extranjeros y mantiene valores similares a los de las cohortes anteriores. Esto se debe a que los porcentajes de exogamia (tanto regional como no regional) se calculan como la proporción de matrimonios con un cónyuge local y el otro no, y no considera en su cálculo a los matrimonios en que ambos integrantes son migrantes. De esta forma se pierde información valiosa, particularmente porque en este último período se celebraron en el Registro Civil de Amboy 14 uniones en las que ambos cónyuges procedían del exterior de Calamuchita, siendo 7 de estos matrimonios entre migrantes extra-continentales. Por otra parte, el índice de exogamia de Palatnik, que se calcula como el porcentaje de progenitores (consortes) de otro lugar sobre el porcentaje de progenitores locales, no presenta esa limitación y recoge toda la información existente. Es por eso que para la sexta cohorte muestra un valor de exogamia que triplica a los de los períodos anteriores. La proporción $\mathrm{m} / \mathrm{f}$ muestra siempre valores mayores a 1 , es decir que los migrantes fueron en todas las cohortes mayoritariamente hombres. Esta particularidad es notoria en el caso de migrantes europeos, ya que sólo el 12\% de ellos fueron mujeres.

En la Tabla 3 se presentan los resultados del análisis de consanguinidad por isonimia. El coeficiente de consanguinidad marital total $(\mathrm{Ft})$ presenta durante la primera cohorte el valor más bajo de todo el período 
Tabla 2. Porcentajes de endogamia y exogamia, índice de exogamia de Palatnik y proporción de migrantes masculinos/femeninos.

Table 2. Inbreeding and exogamy rates, Palatnik exogamy rate, and male/female migrant ratio.

\begin{tabular}{|c|c|c|c|c|c|}
\hline \multicolumn{2}{|c|}{} & \multicolumn{2}{c|}{ Porcentaje de exogamia } & Índice de & Relación \\
\hline Período & $\begin{array}{c}\% \\
\text { endogamia }\end{array}$ & regional & no regional & exogamia & $\boldsymbol{m} / \boldsymbol{f}$ \\
\hline $1896-1900$ & 78,45 & 16,57 & 1,67 & 0,135 & 1,39 \\
\hline $1901-1910$ & 80,67 & 16,62 & 0,3 & 0,122 & 2,04 \\
\hline $1911-1920$ & 79,82 & 15,79 & 4,39 & 0,118 & 1,67 \\
\hline $1921-1930$ & 81,16 & 14,49 & 3,62 & 0,108 & 2,86 \\
\hline $1931-1940$ & 81,61 & 11,49 & 4,02 & 0,119 & 1,64 \\
\hline $1941-1950$ & 58,68 & 28,14 & 4,79 & 0,331 & 1,68 \\
\hline
\end{tabular}

Tabla 3. Análisis de consanguinidad por Isonimia Marital. Fr: componente aleatorio, Fn: componente no aleatorio, Ft: consanguinidad total

Table 3. Analysis of inbreeding by Marital Isonymy. Fr: random component, Fn: non-random component, Ft: total inbreeding.

\begin{tabular}{|c|c|c|c|c|c|}
\hline & \multicolumn{2}{|c|}{ Matrimonios } & \multicolumn{3}{l|}{} \\
\hline Período & Total & Isónimos & Fr & Fn & Ft \\
\hline $1896-1900$ & 181 & 7 & 0,0045 & 0,0053 & 0,0097 \\
\hline $1901-1910$ & 331 & 20 & 0,0037 & 0,0117 & 0,0154 \\
\hline $1911-1920$ & 114 & 16 & 0,0062 & 0,0296 & 0,0356 \\
\hline $1921-1930$ & 138 & 11 & 0,0038 & 0,0164 & 0,0201 \\
\hline $1931-1940$ & 174 & 20 & 0,0065 & 0,0228 & 0,0292 \\
\hline $1941-1950$ & 167 & 11 & 0,0027 & 0,0139 & 0,0166 \\
\hline
\end{tabular}

investigado, estando integrado en partes iguales por los componentes aleatorio (Fr) y no aleatorio (Fn). En las siguientes cohortes se incrementa marcadamente la consanguinidad no aleatoria, particularmente en la tercera y la quinta, donde Fn llega a ser cuatro veces mayor que Fr. En la tercera cohorte aparecen los valores más elevados de consanguinidad. Probablemente, esto se debe a que los casamientos registrados fueron en su mayoría entre personas de esa localidad o bien de lugares muy cercanos. La consanguinidad aleatoria presentó valores muy bajos a lo largo de todo el período investigado, particularmente en la última cohorte.

\section{Discusión}

Los efectos genéticos de la migración se reflejan a través del flujo génico y éste se debe, entre otros fenómenos, al traslado matrimonial, cuya magnitud puede ser apreciada a través de las distancias maritales promedio. En el presente trabajo, los valores más bajos de DMP se dieron en la tercera y cuarta cohortes $(33,7$ y 30,3 km, respectivamente), probablemente como consecuencia de la apertura de nuevos registros civiles en el Departamento Calamuchita, hecho que condujo a una elevada proporción de matrimonios entre personas locales o de lugares próximos a Amboy. En términos comparativos, sin embargo, estas distancias son bastante mayores a la reportada para el Departamento Pocho (Provincia de Córdoba) por Colantonio (1995) para el período 1960-1989, de apenas $10 \mathrm{~km}$, aunque sensiblemente inferiores a las observada en el Valle Calchaquí (68,3 km) por Torres et al. (2019) y en la Puna jujeña $(97,8$ km) por Alfaro y Dipierri (1996). Estos valores se incrementaron notoriamente en la quinta $(58 \mathrm{~km}) \mathrm{y}$, principalmente, en la sexta cohorte $(128 \mathrm{~km})$, debido a la disminución de las uniones locales y al aumento de los matrimonios donde uno de los cónyuges procedía de otros departamentos de Córdoba o de otras provincias argentinas.

La endogamia es un sistema de matrimonios donde la elección del cónyuge se da dentro de un grupo determinado. Luego de varias generaciones, la endogamia conducirá a la consanguinidad (Alfaro, 2010), la cual se duplicará en cada generación en ausencia de flujo génico (Relethford, 2012). Este comportamiento, característico de comunidades pequeñas y aisladas, es resultante de dos situaciones particulares. En primer lugar, puede deberse simplemente a que existe baja diversidad de variantes genéticas debido al aislamiento reproductivo de la población sostenido en el tiempo, hecho que facilita la acción de la deriva génica (Herrera Paz, 2013). La endogamia también puede ser consecuencia de una conducta social pautada por cuestiones religiosas, sociales o económicas. Los resultados de este trabajo muestran, 
Tabla 4. Datos censales para Argentina, Córdoba y el Departamento Calamuchita durante el período investigado.

Table 4. Census data for Argentina, Córdoba and Department of Calamuchita during the period of investigation.

\begin{tabular}{|c|c|c|c|c|c|}
\hline Censo & \multicolumn{3}{|c|}{ Población } & $\begin{array}{c}\text { \#extranjeros } \\
\text { (\%) }\end{array}$ & $\begin{array}{c}\% \\
\text { extranjeros }\end{array}$ \\
\hline Nacional & Argentina & Córdoba & Calamuchita & Calamuchita & Argentina \\
\hline 1895 & 4.044 .911 & 351.223 & 10.647 & $85(0,8)$ & 25,4 \\
\hline 1914 & 7.903 .662 & 735.472 & 12.260 & $1.066(8,6)$ & 29,9 \\
\hline 1947 & 15.893 .827 & 1.497 .987 & 26.684 & $1.764(6,6)$ & 15,3 \\
\hline 2010 & 40.117 .096 & 3.308 .876 & 54.730 & $1.224(2,2)$ & 4,5 \\
\hline
\end{tabular}

Tabla 5. Estimaciones del coeficiente de consanguinidad por isonimia en otras poblaciones históricas y contemporáneas.

Table 5. Estimates of the inbreeding coefficient by isonymy in other historical and contemporary populations.

\begin{tabular}{|l|l|c|c|c|l|}
\hline \multicolumn{1}{|c|}{ Población } & \multicolumn{1}{c|}{ Período } & $\boldsymbol{F}_{\boldsymbol{r}}$ & $\boldsymbol{F}_{\boldsymbol{n}}$ & $\boldsymbol{F}_{\boldsymbol{t}}$ & \multicolumn{1}{c|}{ Referencia } \\
\hline Calamuchita (Córdoba) & $1896-1950$ & 0,0046 & 0,0166 & 0,0211 & Este trabajo \\
\hline Puna (Jujuy) & Actual & 0,0046 & 0,0092 & 0,0139 & Alfaro y Dipierri, 1996 \\
\hline Quebrada (Jujuy) & Actual & 0,0043 & 0,0012 & 0,0055 & Alfaro y Dipierri, 1996 \\
\hline Valle (Jujuy) & Actual & 0,0027 & $-0,0012$ & 0,0015 & Alfaro y Dipierri, 1996 \\
\hline Tulumba (Cordoba) & $1901-1910$ & 0,0023 & 0,0096 & 0,0119 & Kuffer y Colantonio, 2018 \\
\hline Paraguay & Actual & 0,0024 & 0,0017 & 0,0007 & Dipierri et al., 2011 \\
\hline Escazú, Costa Rica & 1890 & 0,0054 & 0,0046 & 0,0101 & Madrigal y Ware, 1997 \\
\hline Abiquiu, New Mexico, EEUU & $1882-1910$ & 0,0117 & 0,0444 & 0,0556 & Devor, 1980 \\
\hline Massachussets, EEUU & 1849 & 0,0025 & 0,0021 & 0,0045 & Relethford y Jaquish, 1988 \\
\hline Val di Non, Italia & $1825-1849$ & 0,009 & 0,0025 & 0,0114 & Gueresi et al., 2001 \\
\hline Andorra & $1601-1951$ & 0,0013 & 0,0018 & 0,0031 & Gonzalez Toja, 2002 \\
\hline La Cabrera, España & $1881-1989$ & 0,005 & 0,015 & 0,02 & Blanco Villegas et al., 2004 \\
\hline
\end{tabular}

particularmente durante las 5 primeras cohortes, un alto grado de endogamia en el Valle de Calamuchita, sensiblemente superior al observado por Colantonio (1995) en el Departamento Pocho, provincia de Córdoba (70\%) y equivalente al reportado por Alfaro y Dipierri (1996) en la Puna jujeña (82,8\%). En la sexta cohorte (1941-1950) se advierte un cambio en la dinámica poblacional a través del aumento de la exogamia debido al mayor número de matrimonios mixtos y foráneos. La población muestra mayor diversidad genética, la cual se ve reflejada en las uniones civiles con personas provenientes de lugares distantes de la región y de Argentina, y también como consecuencia del arribo de un número mayor de inmigrantes europeos.

Hacia finales del siglo XIX y durante la primera mitad del siglo $X X$, Argentina recibió una gran cantidad de población inmigrante, principalmente proveniente de Europa, y en menor medida del Medio Oriente. Ésta constituía el 25 por ciento del total de la población a finales del siglo XIX, y alrededor del 15 por ciento hacia el final de la primera mitad del siglo XX. Estas grandes olas migratorias que comenzaron a fines del siglo XIX se intensificaron entre la Primera y la Segunda Guerra Mundial (Devoto, 2007), en el marco de crisis económicas, sociales, políticas y humanitarias. Sin embargo, los censos nacionales muestran que esta migración masiva no impactó de manera similar en Calamuchita (Tabla 4). De acuerdo a los datos obtenidos de matrimonios celebrados en el Registro Civil de Amboy, el incremento en la proporción de europeos en Calamuchita se advierte recién durante la última cohorte (1941-1950). Es decir que, al menos durante el período investigado, esa población se comportaba como un aislado poblacional, hecho que es confirmado por los altos valores de endogamia observados.

Otro hallazgo que merece ser destacado es que de los 41 cónyuges procedentes de Europa encontrados en el período investigado sólo 5 fueron mujeres (12\%), mostrando un patrón migratorio sexo-asimétrico. Jobling et al. (2004) sostienen que, en general, los hombres tienden a emigrar a distancias más lejanas que las mujeres. Este hecho se ha observado principalmente en las migraciones intercontinentales históricas, donde los migrantes han sido en su mayoría hombres de profesión exploradores, soldados, comerciantes, etc. Estos autores sostienen que para entender el impacto de las migraciones sobre el acervo genético de las poblaciones es necesario también examinar los patrones migratorios locales. Tanto en los medios rural como urbano, las migraciones matrimoniales no se producen al azar, sino 


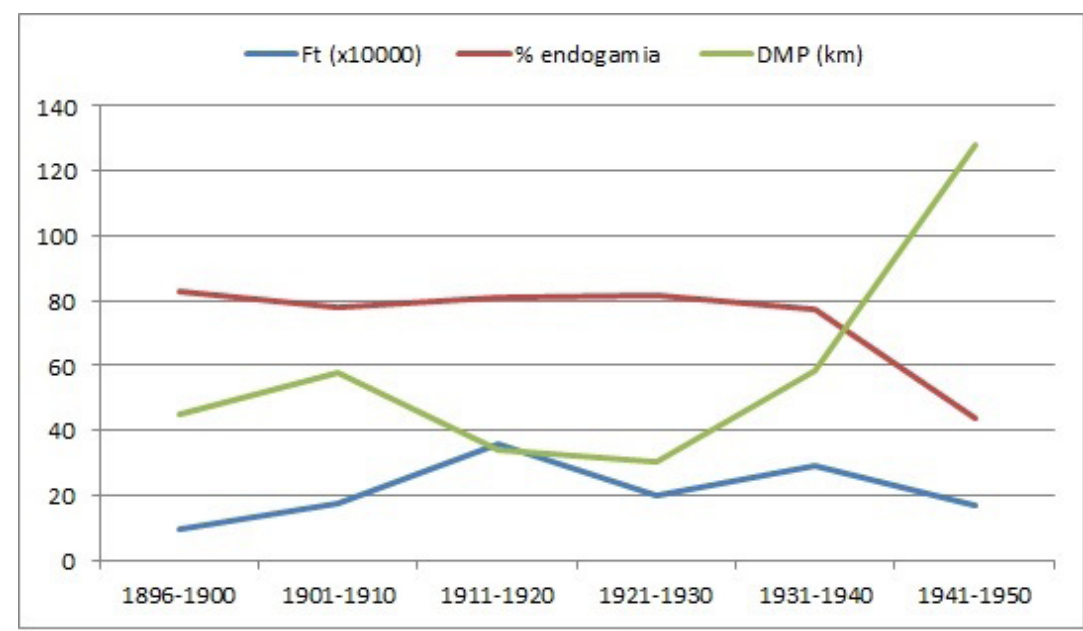

Figura 1. Evolución de la distancia marital promedio (DMP), de la endogamia y del coeficiente de consanguinidad total (Ft) durante el período 1896-1950.

Figure 1. Evolution of the average marital distance (DMP), endogamy and total inbreeding coefficient (Ft) during the period 1896-1950. que, a esa escala, tiene mucha importancia el sistema de convivencia post-matrimonial de la pareja, en términos de patrilocalidad y matrilocalidad. A nivel global, se estima que cerca del $70 \%$ de las poblaciones son patrilocales (Jobling et al., 2004). En Calamuchita, durante el período estudiado, se observa que, de los 1.105 matrimonios registrados, más del 75\% de las mujeres se casó en un el mismo lugar donde nació, mientras que cerca del $40 \%$ de los varones se casaron fuera de su lugar de nacimiento. Este resultado sugiere un patrón de residencia matrilocal y muestra una mayor movilidad masculina, probablemente en busca de fuentes de trabajo y de mejores condiciones económicas. Este resultado coincide con lo encontrado por Torres et al. (2019) en los valles salteños de Lerma y Calchaquí, para los que reportan un claro patrón de residencia matrilocal.

La Tabla 5 muestra estimaciones del coeficiente de consanguinidad por isonimia en otras poblaciones de diferentes contextos históricos y geográficos. La recopilación realizada no pretende ser una revisión exhaustiva de todos los artículos publicados sobre el tema sino más bien una referencia para poder evaluar de forma comparativa los resultados del presente estudio. Esa comparación permite observar que los valores de consanguinidad promedio en la región de Calamuchita durante el período estudiado son realmente elevados, siendo solamente superados por el reportado por Devor (1980) en su estudio acerca de la estructura de matrimonios de la población hispana rural de Abiquiu, ubicada al norte de Nuevo México.

Valores elevados de consanguinidad no aleatoria no son la excepción, habiéndose encontrado, por ejemplo, en la Puna jujeña (Alfaro y Dipierri, 1996) y en La Cabrera, España (Blanco Villegas et al., 2004). Kuffer y Colantonio (2018), en su análisis por isonimia de la población de Tulumba (provincia de Córdoba) a comienzos de los siglos XIX y $\mathrm{XX}$, encontraron el mismo patrón, es decir, casamientos entre linajes familiares mucho más frecuentes que los esperados aleatoriamente. Estos autores sostienen que esos resultados son consecuencia de una estrategia por mantener a salvo el patrimonio familiar en un contexto de oportunidades económicas decrecientes que existía en esa región a comienzos del siglo $X X$, sumado a una posible preferencia conyugal por origen geográfico cercano. Esta interpretación nos parece válida para explicar, al menos parcialmente, los resultados del presente trabajo. Según el registro civil de Amboy, el 65\% de los esposos en matrimonios isónimos eran de profesión "estanciero" o bien hijos de estancieros (es decir, propietarios de tierras). En el caso de las esposas de matrimonios isónimos, el porcentaje de hijas de estancieros ${ }^{4}$ alcanza el $42 \%$ y, en aproximadamente el $25 \%$ de estos matrimonios, ambos cónyuges eran propietarios o hijos de propietarios de tierras. Este porcentaje no resulta aún mayor debido a que en el $35 \%$ de los matrimonios no existe información acerca de la ocupación del padre de las esposa. Otro dato que refuerza esta hipótesis es que en los casamientos isónimos entre terratenientes se repiten los mismos apellidos a través del tiempo (es decir, en las distintas cohortes), siendo Martínez el más frecuente, seguido por Torres y Vélez. Por otra parte, es interesante mencionar que las uniones isónimas no se dan con alta frecuencia solamente entre los terratenientes, con la supuesta finalidad de preservar el patrimonio, sino que también es posible observar una preferencia matrimonial en el otro extremo del entramado social, el integrado por esposos jornaleros y esposas hijas de jornaleros o bien costureras sin información sobre la profesión paterna. En este caso los apellidos más frecuentes son, en primer lugar, Pereyra, seguido por Videla y Medina.

También merece destacarse el hecho de que, a pesar de que la endogamia disminuyó de manera notoria durante la última cohorte (1941-1950), este comportamiento no se refleja en los valores de consanguinidad por isonimia. Si bien la consanguinidad disminuyó levemente durante ese período, no lo hizo

\footnotetext{
4 Recién durante la década de 1930 comienza a consignarse en el registro la ocupación de la esposa.
} 
en la misma proporción que la endogamia o en la que aumentó la distancia marital promedio (Figura 1). Esta inercia en los valores de consanguinidad podría deberse a la persistencia de un comportamiento social, que no fue alterado por el incremento del flujo inmigratorio. Otra interpretación plausible es que, a pesar de que el aislamiento había comenzado a romperse, aún no habría tenido un efecto visible en la estructura genética de la población.

Finalmente, es necesario resaltar la escasa representación de migrantes alemanes, o centro-europeos, encontrados en este estudio. Las actas matrimoniales registran apenas 2 hombres de nacionalidad alemana: uno procedente de Buenos Aires, hijo de terratenientes alemanes radicados en Amboy, quien contrajo matrimonio en 1943 con la hija de un agricultor local, y otro radicado en Villa Rumipal, a $15 \mathrm{~km}$ de Amboy, quien contrajo nupcias en 1947 con una inglesa procedente de Londres. Este resultado, a priori inesperado si se tiene en cuenta la extendida suposición de que Calamuchita constituye una gran comunidad alemana, puede ser, al menos en parte, explicado a través de un breve repaso de la historia reciente del área de estudio. Durante las primeras décadas del periodo abarcado por la presente investigación, la localidad de Villa General Belgrano se denominaba "El Sauce" y figura como el lugar de nacimiento de varias de las personas que se casaron en Amboy. De acuerdo con el relato histórico aceptado por el pueblo, el primer alemán de El Sauce, Pablo Heintze, llegó allí recién en 1930 y, con la intención de instalarse para formar cooperativas agrícolas y forestar de acuerdo al modelo alemán, adquirió dos mil hectáreas, propiedad de un compatriota, Jorge Kappuhn. En 1932 comenzaron a llegar las primeras familias germanas a la zona, el poblado comenzó a crecer y, en 1937, se cambió el nombre de "El Sauce" por "Villa Calamuchita" (Freytes de Vilanova, 2002). En 1940 (durante la Segunda Guerra Mundial), arribaron unos veinte marineros, náufragos del Panzerschiff Admiral Graf Spee, un acorazado alemán hundido en el Río de la Plata. Estos marineros colaboraron con el crecimiento del pueblo, estableciéndose luego mucho de ellos en forma definitiva. Quizá por motivos de disputas entre los criollos y "los gringos", el 9 de julio de 1941 se quemó una bandera argentina, por lo que el Ejecutivo provincial aprobó, como respuesta a esa afrenta, la propuesta para que Villa Calamuchita pasara a denominarse Villa General Belgrano (Freytes de Vilanova, 2002). Por otra parte, Amboy funcionó como el principal registro civil para el Valle de Calamuchita desde 1896 hasta la creación de nuevos registros, algunos mucho más cercanos a Villa General Belgrano, como el de Los Reartes, abierto en 1911 (a 10 km de VGB) y, el de Santa Rosa de Calamuchita (a 12 km de VGB), creado recién en 1943. Es decir, la afluencia masiva de alemanes o de sus descendientes a Calamuchita es, dentro del contexto de esta investigación, relativamente tardía, promediando la década de 1930. Es probable entonces que esta migración comenzara a verse reflejada en las actas matrimoniales de los registros civiles del Departamento Calamuchita aún más tardíamente. Además, es muy probable que estas uniones hayan sido asentadas en el registro civil de Los Reartes primero y más tarde en el de Santa Rosa; queda pendiente corroborar esta hipótesis en un próximo estudio. Por otra parte, resulta evidente que existe un intento por invisibilizar el componente "criollo" mayoritario de la población con el objetivo de promocionar a Villa General Belgrano como una versión local de la "Selva Negra" alemana. Esta intención de mostrar una "hegemonía germánica" es inmediatamente percibida en las fachadas de las calles principales, con fines de explotación turística (Centocchi, 2013). Como ejemplo, ni los medios locales, ni los textos orientados al visitante informan dónde y cuándo se puede disfrutar de eventos tradicionales criollos que aún se desarrollan en la zona, como las carreras cuadreras (Centocchi, 2013).

Córdoba, 2 de diciembre de 2019

\section{Agradecimientos}

Agradecemos a Emma Alfaro y a José Dipierri por el valioso asesoramiento y las múltiples sugerencias, y a Rodrigo Nores por la lectura crítica del manuscrito. Nuestro agradecimiento también a los trabajadores del Archivo del Registro Civil de la Provincia de Córdoba.

\section{Bibliografia}

Alfaro, E. (2010) Dinámica Antroponímica y Estructura Demogenética en Casabindo: Siglos XVI al XXI. Tesis de doctorado inédita. Facultad de Ciencias Naturales y Museo, Universidad Nacional de La Plata.

Alfaro, E. y Dipierri, J.E. (1996) Isonimia, Endogamia, Exogamia y Distancia Marital en la Provincia de Jujuy. Revista Argentina de Antropología Biológica 1(1), 41-56.

Araújo, A.M. y Salzano, F.M. (1974) Marital Distances and inbreeding in Porto Alegre, Brazil. Social Biology 21 (3), 249-255.

Barrai, I., Scapoli, C., Canella, R., Formica, G., Barale, R. y Beretta, M. (1991) Isonymy in Records of Birth and Deaths in Ferrara. Ann. Hum. Biol., 18(5), 95-404.

Blanco Villegas, M.J., Boattini, A., Otero, H.R. y Pettener, D. (2004) Imbreeding patterns in La Cabrera, Spain: Dispensations, multiple consanguinity analysis and isonymy. Human Biology 76(2), 191-210.

Censo 1895. Segundo Censo Nacional. Censos Históricos. Instituto Provincial de Estadística y Censos de la Provincia 
de Santa Fe. Observatorio Económico Social. Universidad Nacional de Rosario.

Censo 1914. Tercer Censo de la República Argentina. Censos Históricos. Instituto Provincial de Estadística y Censos de la Provincia de Santa Fe. Observatorio Económico Social. Universidad Nacional de Rosario.

Censo 1947. Cuarto Censo General de la Nación. Censos Históricos. Instituto Provincial de Estadística y Censos de la Provincia de Santa Fe. Observatorio Económico Social. Universidad Nacional de Rosario.

Centocchi, C.F. (2013) Villa General Belgrano: La germanidad como aglutinante. La trama de la Comunicación 17,115-129.

Colantonio, S.E. (1995) Análisis Histórico-Demográfico de un Semiaislado en la Provincia de Córdoba. Revista Argentina de Antropología Biológica 1(1), 69-85.

Crow, J.F. (1980) The estimation of inbreeding from isonymy. Human Biology 61 (5/6), 949-954.

Crow, J.F., Mange, A.P. (1965) Measurements of Inbreeding from the frequency of Marriages Between Persons of the Same Surnames. Eugenic Quarterly 12,190-203.

Darwin, G.H. (1875) Marriages Between First Cousins in England and their Effects. J. Statis. Soc. 38,153-184.

Demarchi, D.A. y Colantonio, S.E. (2000) Apellidos como alelos de un sistema polimórfico. Ensayo en una población aislada. En: Tendencias Actuales de Investigación en la Antropología Física Española. Caro Dobón, L., Rodriguez Otero, H., Sanchez Compadre Editores. Editorial Universidad de León, España, 265-271.

Devor, E.J. (1980) Marital structure and genetic isolation in a rural hispanic population in northern New Mexico. American Journal of Physical Anthropology 53(2), 257-265.

Devoto, F.J. (2007) La inmigración de ultramar. La migración europea: un fenómeno de larga duración. (Re) Pensar la inmigración en Argentina. Universidad Nacional de Lanús.

Dipierri, J.E. (2014) Apellidos del Noroeste Argentino: Distribución, Isonimia, Estructura y Dinámica Poblacional. Tesis de maestría inédita. Universidad Nacional de Jujuy.

Dipierri, J.E., Rodríguez Larralde, A., Alfaro, E., Andrade, A., Cháves, E. y Barrai, I. (2005) Distribución de Apellidos y Migración En El Noroeste Argentino. Antropo 10, 35-50.

Dipierri, J.E., Rodriguez-Larralde, A. Alfaro, E., Scapoli, C.,
Mamolini, E., Salvatorelli, G., Caramori, G., De Lorenzi, S., Sandri, M., Carrieri, A. y Barrai, I. (2011). A Study of the Population of Paraguay through Isonymy. Annals of Human Genetics 75, 678-687.

Freytes de Vilanova, M. (2002) Aquí me quedo!: Historia de Villa General Belgrano. Editor: Villa General Belgrano, pp. 285.

García, A., Pauro, M., Bravi, C.M., Bailliet, G. y Demarchi, D.A. (2018) Genetic Variation in Populations from Central Argentina Based on Mitochondrial and Y Chromosome DNA Evidences. Journal of Human Genetics 63, 493-507.

Gueresi, S., Pettener, D. y Veronesi, F.M. (2001) Marrige Behaviour in the Alphine Non Valley from 1825 to 1923. Annals of Human Biology 28(2), 157-171.

González Toja, R. (2002) Inbreeding, isonymy and kinstructured migration on the princimality of Andorra. Human Biology 74 (4), 587-600.

Herrera Paz, C. (2013) Apellidos e isonimia en las comunidades garífunas de La Costa Atlántica De Honduras. Estructura Genética e Isonimia. Rev Med Inst Mex. 51(2), 150-157.

INDEC. Censo 2010; Censo general de población y vivienda. Población total, superficie y densidad por provincia. Serie B N² 2 . Tomo 1.

Jobling, M.A., Hurles, M.E. y Tyler-Smith, C. (2004) Human Evolutionary Genetics. Origins, Peoples \& Disease. Garland Science, New York.

Kuffer, C.F. y Colantonio, S.E. (2018) Preferencias conyugales en Tulumba (Córdoba). Comienzos de los siglos XIX y XX. Revista Argentina de Antropología Biológica 20 (1). doi:10.17139/raab.2018.0020.01.03

Lasker, G.W. (1985) Surnames and Genetic Structure. Cambridge Studies in Biological Anthropology. Cambridge University Press.

Lasker, G.W. (1991) Revisión: Datos sobre los apellidos hispanoamericanos en los estudios de biología humana. An. Antrop. (México) 28 (1), 107-128.

Madrigal, L. y Ware, R. (1997) Inbreeding in Escazú, Costa Rica (1800-1840, 1850-1899): isonymy and ecclesiastical dispensations. Human Biology, 69 (5), 703-714.

Palatnik, M. (1975) Estructura poblacional en los tobas del Chaco argentino. Simposio sobre genética de la población Toba del Chaco argentino. En Barbieri, FD, Legname, $\mathrm{AH}$ (Eds): Progresos en Biología. Tucumán: Fundación Miguel Lillo. 
Pauro, M. (2015) Análisis molecular de linajes uniparentales en poblaciones humanas del centro de Argentina. Tesis de doctorado inédita. Facultad de Filosofía y Humanidades, Universidad Nacional de Córdoba.

Rawling, C.P. (1973) A Study of Isonymy. In: Genetic Variation in Britain, Ed. Roberts, DF, Sunderland, E. Taylor and Francis, London, Pp. 83-93.

Relethford, J.H. (2012) Human Population Genetics. WileyBlackwell. New York.

Relethford, J.H. y Jaquish, C.E. (1988) Isonymy, Inbreeding, and Demographic Variation in Historical Massachusetts. American Journal of Physical Anthropology 77 (2), 243-252.
Renaper. Registro Nacional de las personas de la República Argentina.

Rodríguez-Larralde, A. (1990) Distribución de los Apellidos y Su Uso en la Estimación de Aislamiento y Sedentarismo en los Municipios del Estado Lara, Venezuela. Acta Científica Venezolana 41, 163-170.

Tell, S. (2008) Córdoba rural, una sociedad campesina (1750-1850). Prometeo Libros. Buenos Aires, Argentina.

Torres, S.P., Albeza, M.V. y Acreche, N. (2019) Distancias Maritales, Salta (Argentina). Revista Argentina de Antropología Biológica 21 (2), doi:10.24215/18536387e005 